\title{
Correspondence
}

\section{When to do a lumbar puncture in a neonate}

Sir,

We enjoyed Dr Halliday's annotation on neonatal lumbar puncture but felt that his discussion of the role of lumbar puncture in suspected sepsis left an unfortunate question mark hanging over this procedure. ${ }^{\prime}$

It is important we feel to distinguish between early onset and late onset sepsis. In early onset sepsis the usual route of infection is by ascending infection that causes neonatal pneumonia, often clinically and radiologically indistinguishable from hyaline membrane disease, and septicaemia. Frequently babies with group B streptococcal pneumonia are septicaemic at birth, ${ }^{2}$ and as meningitis occurs secondary to high level bacteraemia it is scarcely surprising that about $30 \%$ of babies with early onset group B streptococcal sepsis have meningitis. ${ }^{34} \mathrm{Dr}$ Halliday quotes Eldadah and colleagues' two year study, ${ }^{5}$ which showed only five cases of group B streptococcal pneumonia and septicaemia, none of whom had meningitis, as a reason for not doing early lumbar punctures. Over a five year period (1984-9) with 5000 to 6000 live births per year we have seen seven cases of early onset meningitis $(<48$ hours old): four were due to group B streptococci, and one each to Listeria monocytogenes, Escherichia coli, and Streptococcus mitis. There were no clinical features to distinguish them from septicaemia without meningitis. In two of the seven cases blood cultures were negative. A positive lumbar puncture caused us to revise our antibiotic regime in two cases.

The situation with suspected late onset sepsis is superficially similar but the organisms are different. Over the same five year period, we have seen four cases of Gram negative bacillary meningitis (one pseudomonas, one klebsiella, one achromobacter, and one with both klebsiella and $E$ coli). In all cases the blood cultures were positive, but a positive lumbar puncture altered our antibiotic regime.

It is sometimes argued that if the same antibiotic regime is used to treat late onset septicaemia alone as is used to treat meningitis, then one can rely on blood cultures without performing a lumbar puncture. However, about $10 \%$ of late onset meningitis occurs with negative blood cultures. ${ }^{6}$ If, as is increasingly the case, antibiotics are stopped after two or three days in the face of negative blood cultures, the occasional case of meningitis will be missed and will progress. Although rare, such an outcome would be disastrous. In addition, we have seen two unsuspected cases of Candida albicans meningitis and four cases of enteroviral meningitis over the same five year period. It is important to diagnose the former for early treatment and the latter to prevent cross infection.

We certainly believe that lumbar puncture can lead to significant respiratory deterioration in babies with hyaline membrane disease, particularly those with pulmonary hypertension, and we defer the procedure in such babies. In general, however, we believe that for both early and late onset sepsis, it is important that a lumbar puncture, which is after all a 'biopsy' of cerebrospinal fluid giving instant results, be performed. This affects prognosis and directs antibiotic and supportive treatment appropriately.

\section{IsAACs and S DoBsoN Infectious Diseases Unit, Department of Paediatrics, John Radcliffe Hospital, Headington, Oxford $O X 39 D U$}

Dr Halliday comments:

I thank Drs Isaacs and Dobson for their comments on my annotation. ${ }^{1}$ If a question mark hangs over the role of lumbar puncture in suspected sepsis then I am afraid that the doctors from Oxford have not removed it. Most paediatricians would agree that all babies with early and late onset sepsis should have a lumbar puncture performed, but the problem is in making a diagnosis of suspected sepsis. The Oxford data do not help in this respect, showing a low incidence of early onset meningitis with only seven cases over a five year period. Drs Isaacs and Dobson do not state their indications for doing a lumbar puncture in suspected early onset sepsis but I suspect that many hundreds of babies had this procedure performed to pick up just two babies with meningitis whose blood cultures were negative.

The incidence of late onset meningitis in Oxford is also low with only four cases detected over five years-all of whom had positive blood cultures. While agreeing that about $10 \%$ of late onset meningitis occurs with negative blood cultures ${ }^{6} 7$ this was not so in Oxford so that Drs Isaacs and Dobson would not have stopped antibiotics 'after two or three days in the face of negative blood cultures'. Surely it is unlikely that any paediatrician would stop antibiotics after two or three days in an ill baby who has negative blood cultures?

The picture remains unclear; we know little of the cost benefit ratio of neonatal lumbar puncture for many indications. Neonatologists must be sure that the procedures they undertake to screen selectively populations of neonates are not more hazardous overall than the disease that they are trying to detect.

Perhaps the only way to answer this question is to perform a randomised controlled trial comparing liberal use of lumbar puncture with more restricted indications as suggested in the annotation. Such a trial would have to be multicentre and look carefully at both short and long term outcome. Clinicians who are sure that their own practice is better would of course be excluded from such a study. Until firm evidence is available precise indications for lumbar puncture in the newborn will remain subjective. 